\title{
Analogue of the Theorem of Birkhoff in a Metric Nonsymmetric Theory of the Gravitational-Electromagnetic Field
}

\author{
S. Ragusa ${ }^{1}$ and D. Bosquetti ${ }^{2}$ \\ 1. Instituto de Física de São Carlos, Universidade de São Paulo, C.P. 369, 13560-970 São Carlos, SP, Brazil \\ 2. Faculdade de Tecnologia de Mococa, Mococa, SP, Brazil
}

Received on 6 January, 2007

\begin{abstract}
We prove that a spherically symmetric exterior solution of the field equations of a metric nonsymmetric theory
\end{abstract} of gravitation coupled with the electromagnetic field is necessarily static.

Keywords: Birkhoff; Nonsymmetric; Gravitation; Electromagnetism

\section{INTRODUCTION}

Recently [1] we have shown that the solution of the field equations of a metric nonsymmetric theory of gravitation [2I,II] outside a spherically symmetric time-dependent matter distribution is necessarily static. This is the analogue of the theorem of Birkhoff of general relativity (GR). Here we extend the result to the situation in which electromagnetic field is also present, that is, when the time-dependent source is charged. This is the situation for instance for a radially pulsating charged sphere. The proof of the result, which we will call the static theorem, will run along the same lines of GR as presented, for instance, in [3].

The form of the field equations when the electromagnetic is included into the theory was studied in [4] and the solution for a point charged mass was obtained. In what follows we will be making use of the field equations of that paper.

The paper is organized as follows. In Sec. II we present the vacuum field equations of the theory and in Sec. III, we prove the static theorem. In Sec. IV we draw our conclusions.

\section{THE VACUUM GRAVITO-ELECTROMAGNETIC FIELD EQUATIONS}

When the electromagnetic field is present, the vacuum gravitational field equations of the theory become $[4](\kappa=8 \pi G)$

$$
\begin{gathered}
U_{\alpha \beta}+\Lambda g_{(\alpha \beta)}=\frac{\kappa}{4 \pi} E_{(\alpha \beta)}, \\
\mathbf{g}^{(\alpha \beta)}{ }_{\gamma}+\mathbf{g}^{(\alpha \sigma)} \Gamma_{(\sigma \gamma)}^{\beta}+\mathbf{g}^{(\beta \sigma)} \Gamma_{(\sigma \gamma)}^{\alpha}-\mathbf{g}^{(\alpha \beta)} \Gamma_{(\sigma \gamma)}^{\sigma}=0, \\
\mathbf{g}^{[\alpha \beta]}{ }_{, \beta}=0, \\
\Lambda g_{[\alpha \beta, \gamma]}=\frac{\kappa}{4 \pi} E_{[\alpha \beta, \gamma]}
\end{gathered}
$$

and

$$
\begin{gathered}
\partial_{v}\left\{\sqrt { - g } f \left[g^{(\mu \alpha)} g^{(\nu \beta)}+(2 Z-1) g^{[\mu \alpha]} g^{[\nu \beta]}\right.\right. \\
\left.\left.+(1-Z) g^{[\alpha \beta]} g^{[\mu v]}\right] F_{\alpha \beta}\right\}=0 .
\end{gathered}
$$

We use the notation $a_{(\alpha \beta)}=\left(a_{\alpha \beta}+a_{\beta \alpha}\right) / 2$ and $a_{[\alpha \beta]}=\left(a_{\alpha \beta}-\right.$ $\left.a_{\beta \alpha}\right) / 2$ for the symmetric and antisymmetric parts of $a_{\alpha \beta}$ and the notation $a_{[\alpha \beta, \gamma]}=a_{[\alpha \beta], \gamma}+a_{[\gamma \alpha], \beta}+a_{[\beta \gamma], \alpha}$ for the curl of $a_{[\alpha \beta]}$. In the first equation

$$
U_{\alpha \beta}=\Gamma_{(\alpha \beta), \sigma}^{\sigma}-\Gamma_{(\sigma \alpha), \beta}^{\sigma}+\Gamma_{(\alpha \beta)}^{\rho} \Gamma_{(\rho \sigma)}^{\sigma}-\Gamma_{(\alpha \sigma)}^{\rho} \Gamma_{(\rho \beta)}^{\sigma},
$$

symmetric because the second term is (see (2.14) below) and containing only the symmetric part of the connection, is the analogue of the Ricci tensor. $\Lambda$ is the cosmological constant and

$$
\begin{aligned}
& E_{\alpha \beta}= \frac{1}{4}\left(f g_{\alpha \beta}-2 \frac{\partial f}{\partial g^{\alpha \beta}}\right) g^{\lambda \sigma} g^{\rho \mu}\left(Z F_{\lambda \rho} F_{\sigma \mu}\right. \\
&\left.+(1-Z)\left[F_{\sigma \rho} F_{\lambda \mu}+F_{\lambda \sigma} F_{\rho \mu}\right]\right) \\
&-f g^{\mu \nu}\left(Z F_{\alpha \mu} F_{\beta \nu}+(1-Z)\left[F_{\alpha \nu} F_{\beta \mu}+F_{\mu \nu} F_{\alpha \beta}\right]\right) .
\end{aligned}
$$

Here and in (2.5) $Z$ is an arbitrary parameter. However, the point charge solution turned out to be independent of $Z$. $F_{\mu v}=$ $A_{v, \mu}-A_{\mu, v}$ is the electromagnetic field strength tensor and

$$
f=\frac{\sqrt{-g}}{\sqrt{-g_{s}}},
$$

where $g=\operatorname{det} .\left(g_{\alpha \beta}\right)$ and $g_{s}=\operatorname{det} .\left(g_{(\alpha \beta)}\right) . E_{\alpha \beta}$ is a traceless tensor, $g^{\alpha \beta} E_{\alpha \beta}=0$, because we have the relation [4]

$$
g^{\alpha \beta} \frac{\partial f}{\partial g_{\alpha \beta}}=0
$$

The next two equations involve the symmetric and antisymmetric parts of $\mathbf{g}^{\alpha \beta}=\sqrt{-g} g^{\alpha \beta}$ where $g^{\alpha \beta}$ is the inverse of $g_{\alpha \beta}$ as defined by

$$
g^{\alpha \beta} g_{\alpha \gamma}=g^{\beta \alpha} g_{\gamma \alpha}=\delta_{\gamma}^{\beta} .
$$

The second field equation, (2.2), can be solved for the symmetric part of the connection giving [2-I],

$$
\begin{gathered}
\Gamma_{(\alpha \beta)}^{\sigma}=\frac{1}{2} g^{(\sigma \lambda)}\left(s_{\alpha \lambda, \beta}+s_{\lambda \beta, \alpha}-s_{\alpha \beta, \lambda}\right) \\
\quad+\left(g^{(\sigma \lambda)} s_{\alpha \beta}-\delta_{\alpha}^{\sigma} \delta_{\beta}^{\lambda}-\delta_{\alpha}^{\lambda} \delta_{\beta}^{\sigma}\right) C_{, \lambda} .
\end{gathered}
$$


Here

$$
C=\frac{1}{4} \ln \frac{s}{g}
$$

and $s_{\alpha \beta}$, symmetric and with determinant $s$, is the inverse of $g^{(\alpha \beta)}$ as defined by

$$
s_{\alpha \beta} g^{(\alpha \gamma)}=\delta_{\beta}^{\gamma}
$$

In deriving (2.11) from (2.2) we come across the relation

$$
\Gamma_{(\sigma \alpha)}^{\sigma}=\left(\ln \frac{-g}{\sqrt{-s}}\right), \alpha,
$$

which can be re-obtained from that equation. One then sees that the second term on the right of (2.6) is in fact symmetric. Equation (2.5) is the inhomogeneous Maxwell equation in the presence of the metric nonsymmetric field.

Inside the sources of the field the right-hand side of equations (2.1), (2.3) (2.4) and (2.5) would contain, respectively, the source terms $8 \pi G \bar{T}_{(\alpha \beta)}, 4 \pi \sqrt{-g} S^{\alpha}, 8 \pi G \bar{T}_{[\alpha \beta, \gamma]} / \Lambda$ and $-4 \pi \sqrt{-g} J^{\mu}$ with

$$
\bar{T}_{\alpha \beta}=T_{\alpha \beta}-\frac{1}{2} g_{\alpha \beta} T
$$

$T_{\alpha \beta}$ is the stress tensor and $T=g^{\mu \nu} T_{\mu \nu}, S^{\alpha}$ is the fermion number current density and $J^{\mu}$ is the electromagnetic current density.

\section{SPHERICALLY SYMMETRIC VACUUM SOLUTION:} THE STATIC THEOREM

We will then consider the time-dependent spherically symmetric gravitational-electromagnetic field satisfying the empty-space field equations (2.1)-(2.5). The metric is the same one considered in [1], with the components $g_{00}, g_{11}$ and $g_{[01]}$ depending on $r$ and $t$,

$$
\begin{gathered}
g_{00}=\gamma(r, t)=e^{v(r, t)}, \quad g_{11}=-\alpha(r, t)=-e^{\mu(r, t)}, \\
g_{22}=-r^{2}, \quad g_{33}=-r^{2} \sin ^{2} \theta, \\
g_{01}=-g_{10}=-\omega(r, t)
\end{gathered}
$$

and all other components equal to zero. The non-zero components of the inverse matrix are then

$$
\begin{aligned}
& g^{00}=\frac{\alpha}{\alpha \gamma-\omega^{2}} \quad, \quad g^{11}=-\frac{\gamma}{\alpha \gamma-\omega^{2}}, \\
& g^{22}=-\frac{1}{r^{2}} \quad, \quad g^{33}=-\frac{1}{r^{2} \sin ^{2} \theta}, \\
& g^{01}=-g^{10}=\frac{\omega}{\alpha \gamma-\omega^{2}} .
\end{aligned}
$$

We need also $s_{\alpha \beta}$, whose non-zero components are

$$
\begin{array}{lr}
s_{00}=\frac{\alpha \gamma-\omega^{2}}{\alpha}, & s_{11}=-\frac{\alpha \gamma-\omega^{2}}{\gamma}, \\
s_{22}=-r^{2}, & s_{33}=-r^{2} \sin ^{2} \theta .
\end{array}
$$

The determinants have values

$$
g=-\left(\alpha \gamma-\omega^{2}\right) r^{4} \sin ^{2} \theta
$$

and

$$
s=-\frac{\left(\alpha \gamma-\omega^{2}\right)^{2}}{\alpha \gamma} r^{4} \sin ^{2} \theta .
$$

With these relations equation (2.12) gives

$$
C=\frac{1}{4} \ln \frac{\alpha \gamma-\omega^{2}}{\alpha \gamma} \text {. }
$$

As the sources are spherically symmetric only the radial component of the electric field is present, that is,

$$
F_{01}=E(r . t)
$$

and all other components of $F_{\alpha \beta}$ vanish. Then the non-zero components of the electromagnetic stress tensor are given by [4]

$$
\begin{gathered}
E_{00}=\frac{E^{2}}{2 \alpha}\left(1+\frac{\omega^{2}}{\alpha \gamma}\right)\left(1-\frac{\omega^{2}}{\alpha \gamma}\right)^{-1 / 2}, \\
E_{11}=-\frac{\alpha}{\gamma} E_{00}, \\
E_{22}=\frac{E^{2} r^{2}}{2 \alpha \gamma}\left(1-\frac{\omega^{2}}{\alpha \gamma}\right)^{-1 / 2}, \\
E_{33}=E_{22} \sin ^{2} \theta
\end{gathered}
$$

and

$$
E_{01}=-E_{10}=\frac{\omega E^{2}}{\alpha \gamma}\left(1-\frac{\omega^{2}}{\alpha \gamma}\right)^{-1 / 2} .
$$

Let us see the form acquired by the field equations. From the onset we see, from the last relation in (3.1) and (3.8e), that (2.4) is identically satisfied because it involves only the components $g_{[01]}$ and $E_{[01]}$. We consider now (2.3). Due to the last relation in (3.2) there are only two non-trivial components of that equation, that is, for $\alpha=0$ and $\alpha=1$. They are

$$
\mathbf{g}^{[01]}{ }_{r}=0 ; \mathbf{g}^{[10]}, 0=0 .
$$

From (3.4) and the last relation in Eq. (3.2) we have

$$
\mathbf{g}^{[01]}=\frac{\omega r^{2}}{\sqrt{\alpha \gamma-\omega^{2}}} \sin \theta .
$$


From the equations in (3.9) we see that the quantity

$$
\frac{\omega r^{2}}{\sqrt{\alpha \gamma-\omega^{2}}}=F
$$

is a constant, with respect to both space and time. $F$ is the conserved fermion charge number. From here it follows that the combination

$$
\frac{\alpha \gamma}{\alpha \gamma-\omega^{2}}=1+\frac{F^{2}}{r^{4}}
$$

is time-independent. From this result it follows that the quantity $C$ in (3.6) depends only on $r$. Therefore only $C_{, r}$ survives in equation (2.11). We then have

$$
C_{, \lambda}=\delta_{\lambda r} \frac{F^{2}}{r\left(r^{4}+F^{2}\right)} .
$$

Using these two equations we obtain [4] the following values for the non-zero connection components $\left(a^{\prime}=a_{, r}\right)$,

$$
\begin{aligned}
& \Gamma_{(01)}^{0}=\frac{\gamma^{\prime}}{2 \gamma}+\frac{F^{2}}{r\left(r^{4}+F^{2}\right)}, \\
& \Gamma_{00}^{1}=\frac{\gamma^{\prime}}{2 \alpha}+\frac{\gamma}{\alpha} \frac{F^{2}}{r\left(r^{4}+F^{2}\right)}, \\
& \Gamma_{11}^{1}=\frac{\alpha^{\prime}}{2 \alpha}+\frac{F^{2}}{r\left(r^{4}+F^{2}\right)} \\
& \Gamma_{22}^{1}=-\frac{r}{\alpha}, \\
& \Gamma_{33}^{1}=-\frac{r \sin ^{2} \theta}{\alpha}, \\
& \Gamma_{(12)}^{2}=\Gamma_{(13)}^{3}=\frac{r^{3}}{r^{4}+F^{2}}, \\
& \Gamma_{33}^{2}=-\sin \theta \cos \theta \\
& \Gamma_{(23)}^{3}=\cot \theta
\end{aligned}
$$

and $\left(\dot{a}=a_{, t}\right)$

$$
\Gamma_{00}^{0}=\frac{1}{2} \dot{v}, \quad \Gamma_{11}^{0}=\frac{1}{2} e^{\mu-v} \dot{\mu}, \quad \Gamma_{(01)}^{1}=\frac{1}{2} \dot{\mu} .
$$

These three last relations come from

$$
\begin{aligned}
& \Gamma_{00}^{0}=\frac{1}{2} \frac{\alpha}{\alpha \gamma-\omega^{2}} \frac{\partial}{\partial t} \frac{\gamma\left(\alpha \gamma-\omega^{2}\right)}{\gamma \alpha}, \\
& \Gamma_{11}^{0}=\frac{1}{2} \frac{\alpha}{\alpha \gamma-\omega^{2}} \frac{\partial}{\partial t} \frac{\alpha\left(\alpha \gamma-\omega^{2}\right)}{\alpha \gamma}
\end{aligned}
$$

and

$$
\Gamma_{(01)}^{1}=\frac{1}{2} \frac{\gamma}{\alpha \gamma-\omega^{2}} \frac{\partial}{\partial t} \frac{\alpha\left(\alpha \gamma-\omega^{2}\right)}{\alpha \gamma}
$$

when use is made of (3.12).
Let us see now what are the relations that they will bring about to the field equation (2.1). Putting for short

$$
B=r^{4}+F^{2}
$$

we obtain the following non-trivial four relations, corresponding first to $\alpha=\beta=0$,

$$
\begin{gathered}
\left(\frac{\gamma^{\prime}}{2 \alpha}+\frac{\gamma}{\alpha} \frac{F^{2}}{r B}\right)^{\prime}+\left(\frac{\gamma^{\prime}}{2 \alpha}+\frac{\gamma}{\alpha} \frac{F^{2}}{r B}\right)\left(\frac{\alpha^{\prime}}{2 \alpha}-\frac{\gamma^{\prime}}{2 \gamma}+\frac{2 r^{3}}{B}\right) \\
+\gamma \Lambda-\frac{1}{2} \ddot{\mu}+\frac{1}{4} \dot{v} \dot{\mu}-\frac{1}{4} \dot{\mu}^{2}=\frac{\kappa}{4 \pi} E_{00}
\end{gathered}
$$

then to $\alpha=\beta=1$,

$$
\begin{gathered}
\left(\frac{\gamma^{\prime}}{2 \gamma}+\frac{F^{2}+2 r^{4}}{r B}\right)^{\prime}-\left(\frac{\gamma^{\prime}}{2 \gamma}+\frac{F^{2}}{r B}\right)\left(\frac{\alpha^{\prime}}{2 \alpha}-\frac{\gamma^{\prime}}{2 \gamma}\right) \\
-\frac{2 r^{3}}{B}\left(\frac{\alpha^{\prime}}{2 \alpha}+\frac{F^{2}}{r B}-\frac{r^{3}}{B}\right) \\
+\alpha \Lambda-e^{\mu-v}\left(\frac{1}{2} \ddot{\mu}-\frac{1}{4} \dot{v} \dot{\mu}+\frac{1}{4} \dot{\mu}^{2}\right)=\frac{\kappa}{4 \pi} E_{11},
\end{gathered}
$$

next to $\alpha=\beta=2$,

$$
\begin{gathered}
-\left(\frac{r}{\alpha}\right)^{\prime}-\frac{r}{\alpha}\left(\frac{\gamma^{\prime}}{2 \gamma}+\frac{\alpha^{\prime}}{2 \alpha}+\frac{2 F^{2}}{r B}\right)+1-\Lambda r^{2} \\
=\frac{\kappa}{4 \pi} E_{22}
\end{gathered}
$$

and finally to $\alpha=0$ and $\beta=1$

$$
\frac{r^{3}}{F^{2}+r^{4}} \dot{\mu}=0
$$

because according to equation (3.8e) $E_{(01)}=0$. All these relations reduce to those of GR [3] when $F=0$.

From the equation above we see that

$$
\dot{\mu}=0 \text {. }
$$

Therefore, the additional last terms on the left-hand side of both equations (3.17a) and (3.17b) drop out. As $\mu$ is timeindependent it follows from the second relation in (3.1) that this is so also for $\alpha$, that is, it depends only on $r, \alpha=\alpha(r)$. Multiplying (3.17a) by $\alpha / \gamma$ and adding the result to (3.17b) we see, on account of (3.8b), that the right-hand side will vanish, leading then to the same relation as before [4],

$$
\frac{\gamma^{\prime}}{2 \gamma}+\frac{\alpha^{\prime}}{2 \alpha}=\frac{F^{2}}{r\left(F^{2}+r^{4}\right)} \text {. }
$$


Integration gives

$$
\gamma \alpha=k(t)\left(1+\frac{F^{2}}{r^{4}}\right)^{-1 / 2} .
$$

Therefore,

$$
\gamma(r, t)=k(t) \bar{\gamma}(r)
$$

where

$$
\bar{\gamma}(r)=\frac{1}{\alpha}\left(1+\frac{F^{2}}{r^{4}}\right)^{-1 / 2} .
$$

The fact that $\gamma(r, t)$ can depend on time only through a timedependent factor could be guessed before integration because (3.19) tell us that $\gamma^{\prime} / \gamma$ is time-independent and, consequently, $\gamma$ has to have the form written in (3.21). From this we see that only the time part of the line element $d s^{2}$ will contain a time-dependent coefficient, $\gamma d t^{2}=k(t) \bar{\gamma}(r) d t^{2}$. However, this time-dependent factor can be made equal to unity by a change of the time coordinate. In fact it is sufficient to use the new time coordinate $\bar{t}$ given by

$$
d \bar{t}=\sqrt{k(t)} d t
$$

to obtain the time-independent metric in [4],

$$
d s^{2}=\bar{\gamma}(r) d \bar{t}^{2}-\alpha(r) d r^{2}-r^{2} d \theta^{2}-r^{2} \sin \theta d \phi^{2} .
$$

Starting from here and following the same steps of the beginning of this section, we obtain Eq. (3.11) with $\bar{\gamma}$ replacing $\gamma$,

$$
\frac{\omega r^{2}}{\sqrt{\alpha \bar{\gamma}-\omega^{2}}}=F
$$

This tell us that $\omega$ is also time-independent and given by the same expression as before in [4],

$$
\omega(r)=\frac{r F}{\left(r^{4}+F^{2}\right)^{\frac{3}{4}}} .
$$

This follows from (3.25), which gives

$$
\omega^{2}=\frac{\alpha \bar{\gamma} F^{2}}{r^{4}+F^{2}}
$$

and Eq. (3.22).

We consider now equation (2.5)for the electric field in the redefined metric with $\bar{\gamma}$ replacing $\gamma$. As $g_{s}=-\alpha \bar{\gamma} r^{4} \sin ^{2} \theta$ equation (2.8) gives, recalling (3.4),

$$
f=\sqrt{1-\frac{\omega^{2}}{\alpha \bar{\gamma}}} .
$$

Only two non-trivial relations follows from (2.5), those for $\mu=0$ and $\mu=1$. They are

$$
\frac{\partial}{\partial r}\left(\frac{E^{2} r^{2}}{\sqrt{\alpha \bar{\gamma}}}\right)=0
$$

and

$$
\frac{\partial}{\partial t}\left(\frac{E r^{2}}{\sqrt{\alpha \bar{\gamma}}}\right)=0
$$

¿From this last relation we see that $\partial E / \partial t=0$, meaning that $E$ is time-independent.

Therefore, all quantities, the metric coefficients and the electric field, are independent of time. This proves the static theorem: the exterior spherically symmetric gravitationalelectromagnetic field is necessarily static.

We go now to equation (3.17c) and solve for $\alpha(r)$. Using in $(3.8 \mathrm{c})$ the results in (3.19) and the relation

$$
\left(1-\frac{\omega^{2}}{\alpha \bar{\gamma}}\right)^{-1 / 2}=\left(1+\frac{F^{2}}{r^{4}}\right)^{1 / 2},
$$

that follows from (3.27), we have

$$
E_{22}=\frac{Q^{2}}{2 r^{2}}\left(1+\frac{F^{2}}{r^{4}}\right)^{1 / 2} .
$$

Then taking this into $(3.17 \mathrm{c})$ together with (3.16), we obtain, neglecting the small cosmological constant,

$$
-\left(\frac{r}{\alpha}\right)^{\prime}-\frac{r}{\alpha} \frac{3 F^{2}}{r\left(F^{2}+r^{4}\right)}+1=G \frac{Q^{2}}{r^{4}}\left(1+\frac{r^{4}}{F^{2}}\right)^{1 / 2} .
$$

Choosing the constant of integration equal to $-2 M G$ ( $M$ is the mass of the charged particle) in such a way that the ReissnerNordström (RN) result appears when $F=0$, we obtain, as in [4],

$$
\frac{1}{\alpha(r)}=\left(1+\frac{F^{2}}{r^{4}}\right)-\left(1+\frac{F^{2}}{r^{4}}\right)^{3 / 4}\left(\frac{2 M G}{r}+\frac{G Q^{2}}{r} h(r)\right),
$$

where

$$
h(r)=\int \frac{d r}{r\left(F^{2}+r^{4}\right)^{1 / 4}},
$$

which, as it should, goes to $-r^{-1}$ when $F$ vanishes. Then from (3.22), we obtain

$$
\bar{\gamma}(r)=\left(1+\frac{F^{2}}{r^{4}}\right)^{1 / 2}-\left(1+\frac{F^{2}}{r^{4}}\right)^{1 / 4}\left(\frac{2 M}{r}+\frac{G Q^{2}}{r} h(r)\right) .
$$

Equation (3.35) can be put in the closed form:

$$
\begin{gathered}
h(r)=\frac{1}{2|F|^{1 / 2}}\left[\tan ^{-1}\left(1+\frac{r^{4}}{F^{2}}\right)^{1 / 4}\right. \\
\left.\quad+\frac{1}{2} \ln \frac{\left(1+\frac{r^{4}}{F^{2}}\right)^{1 / 4}-1}{\left(1+\frac{r^{4}}{F^{2}}\right)^{1 / 4}+1}-\frac{\pi}{2}\right],
\end{gathered}
$$

with the term $-\pi / 2$ to give the right limit, $-r^{-1}$, when $F$ vanishes. 
Now the electric field. From (3.29) it then follows that the quantity

$$
\frac{E(r) r^{2}}{\sqrt{\alpha \bar{\gamma}}}=Q
$$

is a constant, the charge of the system. Using (3.22) we have

$$
E(r)=\frac{Q}{r^{2}}\left(1+\frac{F^{2}}{r^{4}}\right)^{-1 / 4} .
$$

This reproduces in fact the point charge $\mathrm{RN}$ result $Q / r^{2}$ for $E$ when $F=0$, that is when $g_{[\alpha \beta]}=0$, the situation in which we recover the Einstein-Maxwell field of GR. At large distances, $r>>|F|^{\frac{1}{2}}, E(r)$ goes into the RN Coulomb field but for small values of $r$, it behaves as $r^{-1}$. So all the results of the time-independent regime are recuperated.
[1] S. Ragusa and D. Bosquetti, Braz. J. Phys. 36, 1223 (2006).

[2] S. Ragusa, Phys. Rev. D 56, 864 (1997). $\Delta$ in this paper is now named $\Gamma$. Also the factor $2 / 3$ in equation (6.7) is dropped here; Gen. Relat. Gravit. 31, 275 (1999). The first term inside the first brackets of equation (23) should be $\gamma / 2 \gamma$. The exponent of $r$ in the expression in the middle of the second line below equation (33) should be 2 instead of 3.These papers will be referred to as I and II, respectively. Equations (1,I-n) and (1,II-n) stand for equations in the corresponding references.

[3] See for instance, A. Papapetrou Lectures on General Relativity (Dordrecht, Holland, 1974).

[4] S. Ragusa and D. Bosquetti, Braz. J. Phys. 30, 569 (2000). 